\title{
On Thermal Convection of a Plasma in Porous Medium
}

\author{
PARDEEP KUMAR*1, SUMIT GUPTA ${ }^{2}$ \\ ${ }^{1}$ Department of Mathematics, ICDEOL, Himachal Pradesh University, Summerhill, \\ Shimla-5, INDIA \\ ${ }^{2}$ Department of Mathematics, Govt. College Arki, Distt Solan (HP), INDIA.
}

\begin{abstract}
The effect of finite Larmor radius of the ions on thermal convection of a plasma is investigated. The case with vertical magnetic field is discussed. Following linear stability theory and normal mode analysis method, the dispersion relation is obtained. It is found that the presence of finite Larmor radius and magnetic field introduces oscillatory modes in the system which were, otherwise, non-existent in their absence. When the instability sets in as stationary convection, finite Larmor radius is found to have a stabilizing effect. Medium permeability has a destabilizing (or stabilizing) effect and the magnetic field has a stabilizing (or destabilizing) effect under certain conditions in the presence of finite Larmor radius effect whereas in the absence of finite Larmor radius effect, the medium permeability and the magnetic field have destabilizing and stabilizing effects, respectively. The sufficient conditions for the non-existence of overstability are also obtained.
\end{abstract}

Key-words: Finite Larmor radius effect, plasma, porous medium, thermal convection, vertical magnetic field

Received: February 19, 2021. Revised: August 5, 2021. Accepted: August 17, 2021. Published: August 31, 2021.

\section{Introduction}

The theoretical and experimental results on thermal convection in a fluid layer, under varying assumptions of hydrodynamics and hydromagnetics, have been discussed in a treatise by Chandrasekhar [1]. The effects of the finiteness of the ion Larmor radius which exhibits itself in the form of 'magnetic viscosity' in the fluid equations have been studied by many authors (Jukes [2]; Vandakurov [3]]) Sharma and Prakash [4] have studied the effect of finite Larmor radius on the thermal instability of a plasma. Melchior and Popowich [5] have considered the finite Larmor radius effect on the Kelvin-Helmholtz instability in a fully ionized plasma while that on Rayleigh-Taylor instability has been studied by Singh and Hans [6]. The effect of finite Larmor radius on the thermal instability of a plasma in the presence of a vertical magnetic field has been studied by Sharma [7].
The study of the breakdown of the stability of a layer of fluid subject to a vertical temperature gradient in porous medium and the possibility of convective flow is of considerable interest in recent years. The study of onset of convection in a porous medium has attracted considerable interest because of its natural occurrence and of its intrinsic importance in many industrial problems, particularly in petroleum-exploration, chemical and nuclear industries. The derivation of the basic equations of a layer of fluid heated from below in porous medium, using Boussinesq approximation, has been given by Joseph [8]. The study of a layer of fluid heated from below in porous media is motivated both theoretically and by its practical applications in engineering disciplines. Among the applications in engineering disciplines one can find the food process industry, chemical process industry, solidification and centrifugal casting of metals. The development of geothermal power resources has increased general interest in the properties of convection in porous medium. Lapwood [9] has studied the stability of convective flow in a porous medium 
using Rayleigh's procedure. The Rayleigh instability of a thermal boundary layer in flow through a porous medium has been considered by Wooding [10].

The properties of ionized space and laboratory magnetic fluids (plasmas) have been intensively investigated theoretically and experimentally in the past sixty years. One of the key aspects studied in this context is the stability of plasma structures. Usually, instabilities can be divided into two categories: macro- and micro-instabilities. Macroinstabilities occur with low frequencies compared to the plasma and cyclotron frequency and they are studied within the framework of magnetohydrodynamics (MHD). Physicists have understood the behavior of macro-instabilities and they showed how to avoid the most destructive of them, but smallscale gradient driven micro-instabilities are still a serious obstacle for having a stable plasma for a large range of parameters. Micro-instabilities are described by models which include, e.g. finite Larmor radius (FLR) and collisionless dissipative effects in plasmas. Time and length scales of micro-instabilities are comparable to the turbulent length scales and the length scales of transport coefficients. In general, the FLR effect is neglected. However, when the Larmor radius becomes comparable to the hydromagnetic length of the problem (e.g. wavelength) or the gyration frequency of ions in the magnetic field is of the same order as the wave frequency, finiteness of the Larmor radius must be taken into account. Strictly speaking, the space and time scale for the breakdown of hydromagnetics are on the respective scales of ion gyration about the field, and the ion Larmor frequency. In the present paper, we explore the effect of FLR on the thermal instability of a plasma in porous medium. Finite Larmor radius effect on plasma instabilities has been the subject of many investigations. In many astrophysical plasma situations such as in solar corona, interstellar and interplanetary plasmas the assumption of zero Larmor radius is not valid. Roberts and Taylor [11] and Rosenbluth et al. [12] have shown the stabilizing influence of finite ion Larmor radius (FLR) effects on plasma instabilities. Hernegger [13] investigated the stabilizing effect of FLR on thermal instability and showed that thermal criterion is changed by FLR for wave propagation perpendicular to the magnetic field. Sharma [14] investigated the stabilizing effect of FLR on thermal instability of rotating plasma. Ariel [15] discussed the stabilizing effect of FLR on thermal instability of conducting plasma layer of finite thickness surrounded by a non-conducting matter. Vaghela and Chhajlani [16] studied the stabilizing effect of FLR on magneto-thermal stability of resistive plasma through a porous medium with thermal conduction. Bhatia and Chhonkar [17] investigated the stabilizing effect of FLR on the instability of a rotating layer of self-gravitating plasma incorporating the effects of viscosity and Hall current. Vyas and Chhajlani [18] pointed out the stabilizing effect of FLR on the thermal instability of magnetized rotating plasma incorporating the effects of viscosity, finite electrical conductivity, porosity and thermal conductivity. Kaothekar and Chhajlani [19] investigated the problem of Jeans instability of self-gravitating rotating radiative plasma with finite Larmor radius corrections. Thus FLR effect is an important factor in the discussion of thermal convection and other hydromagnetic instabilities. Keeping these in mind, an attempt is made to study the thermal convection of incompressible plasma in porous medium in the presence of finite Larmor radius effect in the present paper.

\section{Formulation of the Problem and Perturbation Equations}

Consider an infinite, horizontal, incompressible, viscous and electrically conducting plasma layer of depth $\mathrm{d}$ bounded by the planes $z=0$ and $z=d$ in an isotropic and homogeneous medium of porosity $\varepsilon$ and medium permeability $k_{1}$. This layer is heated from below such that a steady temperature gradient $\quad \beta(=|d T / d z|) \quad$ is maintained. Temperatures and densities at the bottom surface $z=0$ are $T_{0}$ and $\rho_{0}$ and at the upper surface $z=d$ are $T_{1}$ and $\rho_{1}$ respectively. The system is acted on by a uniform vertical magnetic field $\vec{H}(0,0, H)$ and gravity field $\vec{g}(0,0,-g)$. Let $\quad \rho, T, \alpha, \chi, g, \mu_{e}, \mu, v(=$ $\left.\frac{\mu}{\rho_{0}}\right),(u, v, w), \eta$ and $\overleftrightarrow{P}$ denote, respectively, the density, temperature, thermal coefficient of expansion, thermal diffusivity, gravitational 
acceleration, magnetic permeability, viscosity, kinematic viscosity, electrical resistivity and stress tensor taking into account the finite Larmor radius effect. When the plasma flows through a porous medium, the gross effect is represented by Darcy's law. As a result, the resistance term $-\left(\frac{\mu}{k_{1}}\right) \vec{q}$ replaces the usual viscous term, here $\vec{q}$ stands for the filter velocity of the plasma. Then the equations expressing the conservation of momentum, mass and heat are

$$
\begin{aligned}
& \frac{1}{\varepsilon}\left[\frac{\partial \vec{q}}{\partial t}+\frac{1}{\varepsilon}(\vec{q} \cdot \nabla) \vec{q}\right]=-\frac{1}{\rho_{0}} \overleftrightarrow{P}+\vec{g}\left(1+\frac{\delta \rho}{\rho_{0}}\right) \\
& +\frac{\mu_{e}}{4 \pi \rho_{0}}(\nabla \times \overrightarrow{\mathrm{H}}) \times \vec{H}-\frac{v}{k_{1}} \vec{q} \\
& \quad \nabla \cdot \vec{q}=0 \\
& E \frac{\partial T}{\partial t}+(\vec{q} \cdot \nabla) T=\kappa \nabla^{2} T
\end{aligned}
$$

where $E=\varepsilon+(1-\varepsilon) \frac{\rho_{s} C_{s}}{\rho_{0} C} \mathrm{~s} ; \rho_{0}, C$ are the density and heat capacity of the plasma and $\rho_{S}, C_{S}$ are the density and the heat capacity of the solid matrix respectively.

The equation of the state is

$$
\rho=\rho_{0}\left[1-\alpha\left(T-T_{0}\right)\right],
$$

where the suffix zero refers to the values at the reference level $\quad z=0$.

The Maxwell's equations yield

$$
\begin{aligned}
\varepsilon \frac{d \vec{H}}{d t} & =(\vec{H} \cdot \nabla) \vec{q}+\epsilon \eta \nabla^{2} \vec{H}, \\
\nabla . \vec{H} & =0,
\end{aligned}
$$

where $d / d t=\partial / \partial t+(1 / \varepsilon)(\vec{q} \cdot \nabla)$ stands for the convective derivative.

For the magnetic field along the $z$-axis, the stress tensor $\overleftrightarrow{P}$, taking into account the finite ion gyration radius (Vandakurov, [3]), has the components

$$
\begin{gathered}
P_{x x}=p-\rho_{0} v_{0}\left(\frac{\partial u}{\partial y}+\frac{\partial v}{\partial x}\right), \\
P_{x y}=P_{y x}=\rho_{0} v_{0}\left(\frac{\partial u}{\partial x}-\frac{\partial v}{\partial y}\right),
\end{gathered}
$$

$$
\begin{aligned}
& P_{x z}=P_{z x}=-2 \rho_{0} v_{0}\left(\frac{\partial v}{\partial z}+\frac{\partial w}{\partial y}\right), \\
& P_{y y}=p+\rho_{0} v_{0}\left(\frac{\partial u}{\partial y}+\frac{\partial v}{\partial x}\right), \\
& P_{y z}=P_{z y}=2 \rho_{0} v_{0}\left(\frac{\partial w}{\partial x}+\frac{\partial u}{\partial z}\right), \quad P_{z z}=p,
\end{aligned}
$$

where $p$ is the scalar part of the pressure and $\rho_{0} v_{0}=N k^{*} T / 4 \omega_{H}, \omega_{H}$ being the iongyration frequency, $k^{*}$ is Boltzman constant, while $N$ and $T$ are number density and temperature of the ions, respectively.

The steady state solution is

$$
\begin{array}{r}
\vec{q}=(0,0,0), T=T_{0}-\beta z, \quad \rho \\
=\rho_{0}(1+\alpha \beta z),
\end{array}
$$

where $\beta=\frac{T_{0}-T_{1}}{d}$ is the magnitude of uniform temperature gradient which is maintained and is positive as temperature decreases upwards.

Now we consider a small perturbation on the steady state and let $\delta \rho, \delta \overleftrightarrow{P}, \theta, \vec{q}(u, v, w)$ and $\vec{h}\left(h_{x}, h_{y}, h_{z}\right)$ denote, respectively, the perturbations in density, stress tensor, temperature, hydromagnetic fluid velocity (initially zero) and the magnetic field. The change in density $\delta \rho$, caused by the perturbations $\theta$ in temperature, is given by

$$
\delta \rho=-\alpha \rho_{0} \theta .
$$

Then the linearized hydromagnetic perturbation equations relevant to the problem are

$$
\begin{aligned}
\frac{1}{\varepsilon} \frac{\partial \vec{q}}{\partial t}=-\frac{1}{\rho_{0}} \nabla \delta & \overleftrightarrow{P}+\vec{g}\left(\frac{\delta \rho}{\rho_{0}}\right) \quad+\frac{\mu_{e}}{4 \pi \rho_{0}}(\nabla \times \vec{h}) \\
& \times \vec{H} \\
& -\frac{v}{k_{1}} \vec{q}
\end{aligned}
$$

$\nabla \cdot \vec{q}=0$,

$E \frac{\partial \theta}{\partial t}=\beta w+\kappa \nabla^{2} \theta$,

$\varepsilon \frac{\partial \vec{h}}{\partial t}=(\vec{H} \cdot \nabla) \vec{q}+\epsilon \eta \nabla^{2} \vec{h}$,

$\nabla \cdot \vec{h}=0$. 
Within the framework of the Boussinesq approximation, equations (9) - (13) become

$$
\begin{aligned}
& \left(\frac{\partial}{\partial t}+\frac{v \varepsilon}{k_{1}}\right) \nabla^{2}=\varepsilon g \alpha\left(\frac{\partial^{2}}{\partial x^{2}}+\frac{\partial^{2}}{\partial y^{2}}\right) \theta \\
& +\frac{\mu_{e} H \varepsilon}{4 \pi \rho_{0}} \frac{\partial}{\partial z}\left(\nabla^{2} h_{z}\right)+\varepsilon v_{0}\left(\nabla^{2}-3 \frac{\partial^{2}}{\partial z^{2}}\right) \frac{\partial \zeta}{\partial z},
\end{aligned}
$$

$$
\begin{aligned}
\left(\frac{\partial}{\partial t}+\frac{v \varepsilon}{k_{1}}\right) \zeta=\frac{\mu_{e} \varepsilon H}{4 \pi \rho_{0}} \frac{\partial \xi}{\partial z}-\varepsilon v_{0} \\
\left(\nabla^{2}-3 \frac{\partial^{2}}{\partial z^{2}}\right) \frac{\partial w}{\partial z}
\end{aligned}
$$$$
\varepsilon \frac{\partial \xi}{\partial t}=H \frac{\partial \zeta}{\partial z}+\varepsilon \eta \nabla^{2} \xi
$$$$
\left[E \frac{\partial}{\partial t}-\kappa \nabla^{2}\right] \theta=\beta w
$$$$
\varepsilon\left[\frac{\partial}{\partial t}-\eta \nabla^{2}\right] h_{z}=H \frac{\partial w}{\partial z},
$$

where $\nabla^{2}=\partial^{2} / \partial x^{2}+\partial^{2} / \partial y^{2}+\partial^{2} / \partial z^{2}$

and $\quad \zeta=\partial v / \partial x-\partial u / \partial y ; \xi=\partial h_{y} / \partial x-$ $\partial h_{x} / \partial y$

stand for the z-components of vorticity and current density, respectively.

\section{Dispersion Relation}

We decompose the disturbances into normal modes and assume that the perturbed quantities are of the form

$$
\begin{aligned}
& {\left[w, h_{z}, \zeta, \xi, \theta\right]} \\
& =[W(z), K(z), Z(z), X(z), \Theta(z)] \exp \left[i k_{x} x\right. \\
& \left.+i k_{y} y+n t\right],
\end{aligned}
$$

where $k_{x}$ and $k_{y}$ are the wave numbers along the $x-$ and $y-$ directions, respectively, $k=$ $\sqrt{k_{x}^{2}+k_{y}^{2}}$ is the resultant wave number, and $n$ is, in general, a complex constant.

Using expression (19), equations (14) - (18) in non-dimensional form transform to

$$
\begin{array}{r}
\left(\frac{\sigma}{\varepsilon}+\frac{1}{P_{l}}\right)\left(D^{2}-a^{2}\right) W+\left(\frac{g d^{2}}{v}\right) a^{2} \alpha \Theta \\
-\frac{\mu_{e} H d}{4 \pi \rho_{0} v}\left(D^{2}-a^{2}\right) D K \\
+\left(\frac{v_{0} d}{v}\right)\left(2 D^{2}+a^{2}\right) D Z \\
=0 \\
\left(\frac{\sigma}{\varepsilon}+\frac{1}{P_{l}}\right) Z=\left(\frac{\mu_{e} H d}{4 \pi \rho_{0} v}\right) D X \\
+\left(\frac{v_{0}}{v d}\right)\left(2 D^{2}+a^{2}\right) D W \\
{\left[D^{2}-a^{2}-E p_{1} \sigma\right] \Theta=-\left(\frac{\beta d^{2}}{\kappa}\right) W} \\
{\left[D^{2}-a^{2}-p_{2} \sigma\right] K=-\left(\frac{H d}{\varepsilon \eta}\right) D W} \\
{\left[D^{2}-a^{2}-p_{2} \sigma\right] X=-\left(\frac{H d}{\varepsilon \eta}\right) D Z}
\end{array}
$$

where we have introduced new coordinates $\left(x^{*}, y^{*}, z^{*}\right)=(x / d, y / d, z / d)$ in the new unit of length $d$ and $D=d / d z^{*}$. For convenience, the asterisks are dropped hereafter. Also we have put $a=k d$ and $\sigma=$ $n d^{2} / \nu, p_{1}=v / \kappa$ is the Prandtl number, $p_{2}=$ $v / \eta$ is the magnetic Prandtl number and $P_{l}=$ $k_{1} / d^{2}$ is the dimensionless medium permeability.

Here we consider the case where both boundaries are free as well as perfect conductors of heat, while the adjoining medium is electrically non-conducting. The case of two free boundaries is slightly artificial, except in stellar atmospheres (Spiegel [20]) and in certain geophysical situations where it is most appropriate. However, the case of two free boundaries allows us to obtain analytical solution without affecting the essential features of the problem. The appropriate boundary conditions, with respect to which equations (20) - (24) must be solved, are

$$
\begin{aligned}
& W=D^{2} W=0, D Z=0, \Theta=0, X=0 \\
& =0 \text { at } z=0 \text { and } 1,
\end{aligned}
$$

on a perfectly conducting boundary. 
In the absence of any surface current, the tangential components of the magnetic field are continuous. Hence the boundary conditions, in addition to (25), are

$D K=0$ on the boundaries.

Using the above boundary conditions, it can be shown that all the even order derivatives of $W$ must vanish for $z=0$ and 1 and hence the proper solution of $W$ characterizing the lowest mode is

$$
W=W_{0} \sin \pi z,
$$

where $W_{0}$ is a constant.

Eliminating $Z, K, X$ and $\Theta$ between equations (20) - (24) and substituting the proper solution (27) in the resultant equation, we obtain the dispersion relation

$$
\begin{aligned}
& R_{1} x \\
& =(1+x)\left(\frac{i \sigma_{1}}{\varepsilon}+\frac{1}{P}\right)\left(1+x+i E p_{1} \sigma_{1}\right) \\
& +Q_{1} \frac{(1+x)\left(1+x+i E p_{1} \sigma_{1}\right)}{\varepsilon\left(1+x+i p_{2} \sigma_{1}\right)} \\
& +\frac{(x-2)^{2}\left(1+x+i p_{2} \sigma_{1}\right)}{\left[\left(1+x+i E p_{1} \sigma_{1}\right)\right.} \\
& {\left[\left(1+x+i p_{2} \sigma_{1}\right)\left(\frac{i \sigma_{1}}{\varepsilon}+\frac{1}{P}\right)+\frac{Q_{1}}{\varepsilon}\right]}
\end{aligned}
$$

where

$$
Q=\frac{\mu_{e} H^{2} d^{2}}{4 \pi \rho_{0} v \eta}, R=\frac{g \alpha \beta d^{4}}{v \kappa}, U=\frac{v_{0}^{2}}{v^{2}},
$$

stand for the Chandrasekhar number, the thermal Rayleigh number, a non-dimensional number accounting for the finite Larmor radius, respectively, and we have also put

$$
\begin{gathered}
x=\frac{a^{2}}{\pi^{2}}, P=\pi^{2} P_{l}, i \sigma_{1}=\frac{\sigma}{\pi^{2}}, Q_{1}=\frac{Q}{\pi^{2}}, R_{1} \\
=\frac{R}{\pi^{4}} \text { and } i=\sqrt{-1} .
\end{gathered}
$$

\section{Important Theorems and Discussion}

Theorem 1: The system is stable or unstable.

Proof: Multiplying equation (20) by $W^{*}$, the complex conjugate of $W$, and using equations (21) - (24) together with the boundary conditions (25) and (26), we obtain

$$
\begin{aligned}
& \left(\frac{\sigma}{\varepsilon}+\frac{1}{P_{l}}\right) I_{1}-\frac{g \alpha \kappa a^{2}}{v \beta}\left(I_{2}+E p_{1} \sigma^{*} I_{3}\right) \\
& +\frac{\mu_{e} \eta \varepsilon}{4 \pi \rho_{0} v}\left(I_{4}+p_{2} \sigma^{*} I_{5}\right)+d^{2}\left(\frac{\sigma^{*}}{\varepsilon}+\frac{1}{P_{l}}\right) I_{6} \\
& +\frac{\mu_{e} \eta d^{2} \varepsilon}{4 \pi \rho_{0} v}\left(I_{7}+p_{2} \sigma I_{8}\right) \\
& =0
\end{aligned}
$$

where

$$
\begin{gathered}
I_{1}=\int_{0}^{1}\left(|D W|^{2}+a^{2}|W|^{2}\right) d z \\
I_{2}=\int_{0}^{1}\left(|D \Theta|^{2}+a^{2}|\Theta|^{2}\right) d z \\
I_{3}=\int_{0}^{1}\left(|\Theta|^{2}\right) d z \\
I_{4}=\int_{0}^{1}\left(\left|D^{2} K\right|^{2}+2 a^{2}|D K|^{2}+a^{4}|K|^{2}\right) d z \\
I_{5}=\int_{0}^{1}\left(|D K|^{2}+a^{2}|K|^{2}\right) d z \\
I_{6}=\int_{0}^{1}\left(|Z|^{2}\right) d z \\
I_{7}=\int_{0}^{1}\left(|D X|^{2}+a^{2}|X|^{2}\right) d z \\
I_{8}=\int_{0}^{1}\left(|X|^{2}\right) d z .
\end{gathered}
$$

and $\sigma^{*}$ is the complex conjugate of $\sigma$. The integrals $I_{1}, \ldots \ldots \ldots . I_{8}$ are all positive definite. Putting $\sigma=\sigma_{r}+i \sigma_{i}$ and equating the real and imaginary parts of equation (29), we obtain

$$
\begin{gathered}
{\left[\frac{I_{1}}{\varepsilon}+\frac{\mu_{e} \eta \varepsilon p_{2}}{4 \pi \rho_{0} v}\left(I_{5}+d^{2} I_{8}\right)-\frac{g \alpha \kappa a^{2}}{v \beta} E p_{1} I_{3}\right.} \\
\left.+\frac{d^{2}}{\varepsilon} I_{6}\right] \sigma_{r}= \\
-\left[\frac{1}{P_{l}} I_{1}+\frac{\mu_{e} \eta \varepsilon}{4 \pi \rho_{0} v}\left(I_{4}+d^{2} I_{7}\right)-\frac{g \alpha \kappa a^{2}}{v \beta} I_{2}\right. \\
\left.+\frac{d^{2}}{P_{l}} I_{6}\right]
\end{gathered}
$$

and 
$\left[\frac{I_{1}}{\varepsilon}+\frac{g \alpha \kappa a^{2}}{v \beta} E p_{1} I_{3}-\frac{\mu_{e} \eta \varepsilon p_{2}}{4 \pi \rho_{0} v}\left(I_{5}-d^{2} I_{8}\right)\right.$

$\left.-\frac{d^{2}}{\varepsilon} I_{6}\right] \sigma_{i}=0$.

It is evident from equation (31) that $\sigma_{r}$ may be positive or negative. The system is, therefore, stable or unstable.

Theorem 2: The modes may be oscillatory or non-oscillatory in contrast to case of no magnetic field, and in the absence of finite Larmor radius and stable solute gradient where modes are non-oscillatory.

Proof: Equation (32) yields that $\sigma_{i}$ may be zero or non-zero, which means that the modes may be non-oscillatory or oscillatory. In the absence of finite Larmor radius and magnetic field, equation (32) reduces to

$\sigma_{i}\left[\frac{I_{1}}{\varepsilon}+\frac{g \alpha \kappa a^{2}}{v \beta} E p_{1} I_{3}\right]=0$,

and the terms in brackets are positive definite. Thus $\sigma_{i}=0$, which means that oscillatory modes are not allowed and the principle of exchange of stabilities is valid. The oscillatory modes are introduced due to the presence of magnetic field and finite Larmor radius which were non-existent in their absence.

Theorem 3: The system is stable for $\frac{g \alpha \kappa}{v \beta} \leq$ $\frac{27 \pi^{4}}{4}$ and under the condition $\frac{g \alpha \kappa}{v \beta}>\frac{27 \pi^{4}}{4}$, the system becomes unstable.

Proof: From equation (32), it is clear that $\sigma_{i}$ is zero when the quantity multiplying it is not zero and arbitrary when this quantity is zero.

If $\sigma_{i} \neq 0$, then equation (31) gives

$$
\begin{aligned}
\frac{I_{1}}{\varepsilon}+\frac{\mu_{e} \eta d^{2} \varepsilon}{4 \pi \rho_{0} v} p_{2} I_{8} & \\
& =\frac{\mu_{e} \eta \varepsilon}{4 \pi \rho_{0} v} p_{2} I_{5}+\frac{d^{2}}{\varepsilon} I_{6} \\
& -\frac{g \alpha \kappa a^{2}}{v \beta} E p_{1} I_{3}
\end{aligned}
$$

Substituting this in equation (30), we get

$$
\begin{gathered}
\frac{2 \sigma_{r} I_{1}}{\varepsilon}+\frac{1}{P_{l}} I_{1}+\frac{\mu_{e} \eta \varepsilon}{4 \pi \rho_{0} v} I_{4}+d^{2}\left(\frac{1}{P_{l}}\right) I_{6} \\
+\frac{\mu_{e} \eta d^{2} \varepsilon}{4 \pi \rho_{0} v} I_{7} \\
+\frac{2 \sigma_{r} \mu_{e} \eta d^{2} \varepsilon}{4 \pi \rho_{0} v} p_{2} I_{8} \\
=\frac{g \alpha \kappa a^{2}}{v \beta} I_{2} .
\end{gathered}
$$

Equation (34) on using Rayleigh-Ritz inequality gives

$$
\begin{aligned}
& \frac{\left(\pi^{2}+a^{2}\right)^{3}}{a^{2}} \int_{0}^{1}|W|^{2} d z \\
& +\frac{\left(\pi^{2}+a^{2}\right)}{a^{2}}\left\{\frac{2 \sigma_{r} \mu_{e} \eta d^{2} \varepsilon}{4 \pi \rho_{0} v} p_{2} I_{8}+\frac{\mu_{e} \eta d^{2} \varepsilon}{4 \pi \rho_{0} v} I_{7}\right. \\
& \left.+d^{2}\left(\frac{1}{P_{l}}\right) I_{6}+\frac{\mu_{e} \eta \varepsilon}{4 \pi \rho_{0} v} I_{4}+\frac{1}{P_{;}} I_{1}+\frac{2 \sigma_{r}}{\varepsilon} I_{1}\right\} \\
& \leq \frac{g \alpha \kappa}{\beta v} \int_{0}^{1}|W|^{2} d z .
\end{aligned}
$$

Therefore, it follows from equation (35) that

$$
\begin{aligned}
& {\left[\frac{27 \pi^{4}}{4}-\frac{g \alpha \kappa}{v \beta}\right] \int_{0}^{1}|W|^{2} d z} \\
& +\frac{\left(\pi^{2}+a^{2}\right)}{a^{2}}\left\{\frac{2 \sigma_{r} \mu_{e} \eta d^{2} \varepsilon}{4 \pi \rho_{0} v} p_{2} I_{8}+\frac{\mu_{e} \eta d^{2} \varepsilon}{4 \pi \rho_{0} v} I_{7}\right. \\
& \left.+d^{2}\left(\frac{1}{P_{l}}\right) I_{6}+\frac{\mu_{e} \eta \varepsilon}{4 \pi \rho_{0} v} I_{4}+\frac{1}{P_{;}} I_{1}+\frac{2 \sigma_{r}}{\varepsilon} I_{1}\right\} \\
& \leq 0
\end{aligned}
$$

since minimum value of $\frac{\left(\pi^{2}+a^{2}\right)^{3}}{a^{2}}$ with respect to $a^{2}$ is $\frac{27 \pi^{4}}{4}$.

Now, let $\sigma_{r} \geq 0$, we necessary have from (36) that

$$
\frac{g \alpha \kappa}{v \beta}>\frac{27 \pi^{4}}{4}
$$

Hence, if

$$
\frac{g \alpha \kappa}{v \beta} \leq \frac{27 \pi^{4}}{4}
$$

then $\sigma_{r}<0$. Therefore, the system is stable. Thus, under condition (38), the system is stable and under condition (37) the system becomes unstable. 
Theorem 4: For stationary convection case:

(I) Finite Larmor radius has stabilizing effects on the system.

(II) In the absence of finite Larmor radius effect, the medium permeability and the magnetic field have destabilizing and stabilizing effects respectively.

(III) In the presence of finite Larmor radius effect if

$$
\begin{gathered}
{\left[\frac{1+x}{P}+\frac{Q_{1}}{\varepsilon}\right]^{2}>(\text { or }<)} \\
U(x-2)^{2}(1+x)
\end{gathered}
$$

the medium permeability has a destabilizing (or stabilizing) and the magnetic field has a stabilizing (or destabilizing) effect on the system.

Proof: When the instability sets in as stationary convection, the marginal state will be characterized by $\sigma=0$. Putting $\sigma=0$, the dispersion relation (28) reduces to

$$
\begin{aligned}
& =\left(\frac{1+x}{x}\right)\left[\frac{1+x}{P}+\frac{Q_{1}}{\varepsilon}\right] \\
& +\frac{U(x-2)^{2}(1+x)^{2}}{x\left[\frac{1+x}{P}+\frac{Q_{1}}{\varepsilon}\right]},
\end{aligned}
$$

which expresses the modified Rayleigh number $R_{1}$ as a function of the dimensionless wave number $x$ and the parameters $Q_{1}, U$ and $P$. To study the effects of finite Larmor radius, medium permeability and magnetic field on $R_{1}$, we examine the nature of $d R_{1} / d U, d R_{1} / d P$ and $d R_{1} / d Q_{1}$ analytically.

(I) It follows from equation (39),

$$
\frac{d R_{1}}{d U}=\frac{(x-2)^{2}(1+x)^{2}}{x\left[\frac{1+x}{P}+\frac{Q_{1}}{\varepsilon}\right]}>0,
$$

which implies that finite Larmor radius has stabilizing effect on the system.

(II) Also equation (39) yields

$$
\frac{d R_{1}}{d P}=-\frac{(1+x)}{x P^{2}}
$$

$$
\left[\frac{\left(\frac{1+x}{P}+\frac{Q_{1}}{\varepsilon}\right)^{2}-U(x-2)^{2}}{(1+x)}\right]
$$

and

$$
\frac{d R_{1}}{d Q_{1}}=\left(\frac{1+x}{\varepsilon x}\right)
$$

$$
\left[\frac{\left(\frac{1+x}{P}+\frac{Q_{1}}{\varepsilon}\right)^{2}-U(x-2)^{2}(1+x)}{\left(\frac{1+x}{P}+\frac{Q_{1}}{\varepsilon}\right)^{2}}\right]
$$

If

$$
\left[\frac{1+x}{P}+\frac{Q_{1}}{\varepsilon}\right]^{2}<U(x-2)^{2}(1+x),
$$

then

$$
\frac{d R_{1}}{d P}>0 \text { and } \frac{d R_{1}}{d Q_{1}}<0,
$$

exhibiting the stabilizing effect of medium permeability and destabilizing effect of magnetic field respectively.

If

$$
\left[\frac{1+x}{P}+\frac{Q_{1}}{\varepsilon}\right]^{2}>U(x-2)^{2}(1+x),
$$

then

$$
\frac{d R_{1}}{d P}<0 \text { and } \frac{d R_{1}}{d Q_{1}}>0,
$$

meaning thereby the destabilizing effect of medium permeability and stabilizing effect of the magnetic field on the thermal instability of a plasma in porous media.

In the absence of finite Larmor radius effect, equation (41) reduces to

$\frac{d R_{1}}{d P}=-\frac{(1+x)^{2}}{x P^{2}}<0$,

and

$\frac{d R_{1}}{d Q_{1}}=\left(\frac{1+x}{\varepsilon x}\right)>0$.

The medium permeability and magnetic field thus have destabilizing and stabilizing effects respectively. 
Theorem 5: The sufficient conditions for the non-existence of overstability are

$$
\begin{aligned}
& \kappa<\left[\varepsilon+(1-\varepsilon) \frac{\rho_{S} C_{S}}{\rho_{0} C}\right] \eta \text { and } \kappa \\
& <\frac{\left[\varepsilon+(1-\varepsilon) \frac{\rho_{S} C_{S}}{\rho_{0} C}\right] \eta}{P^{3}\left[\left(\frac{v_{0}}{v}\right)^{2} \varepsilon(b-3)^{2}-\frac{\mu_{e} H^{2} d^{2}}{2 \varepsilon^{2} \rho_{0} v \eta \pi^{3} P}\right]} .
\end{aligned}
$$

Proof: Here we discuss the possibility of whether instability may occur as overstability. Since for overstability, our interest is to determine the critical Rayleigh number for the onset of instability via a state of pure oscillations, it will suffice to find conditions for which equation (28) will admit of solutions with $\sigma_{1}$ real.

Assuming that $\sigma_{1}$ is real and equating the real and imaginary parts of equation (28) and eliminating $R_{1}$ between them, we obtain

$$
A_{3} C_{1}^{3}+A_{2} C_{1}^{2}+A_{1} C_{1}+A_{0}=0,
$$

where $C_{1}=\sigma_{1}^{2}, b=1+x$ and

$$
\begin{aligned}
A_{3}=\frac{b p_{2}^{4}}{\varepsilon^{2}}\left(\frac{E p_{1}}{P}\right. & \left.+\frac{b}{\varepsilon}\right) \\
A_{0}=\frac{b^{6}}{\varepsilon P^{2}}+\left[\left(\frac{E p_{1}}{P^{3}}+\frac{2 Q_{1}}{\varepsilon^{2} P}\right)\right. & \left.-\frac{U(b-3)^{2}}{\varepsilon}\right] b^{5} \\
& +\left[\frac{Q_{1}^{2}}{\varepsilon^{3}}+\frac{Q_{1}}{\varepsilon P^{2}}\left(3 E p_{1}-p_{2}\right)\right. \\
& \left.+U(b-3)^{2} \frac{E p_{1}}{P}\right] b^{4} \\
& +\left[\frac{Q_{1}^{2}}{\varepsilon^{2} P}\left(3 E p_{1}-2 p_{2}\right)\right. \\
& +U(b-3)^{2}\left(\frac{p_{2} Q_{1}}{\varepsilon}\right. \\
& \left.\left.+\frac{E p_{1} Q_{1}}{\varepsilon}\right)\right] b^{3} \\
& +\frac{Q_{1}^{3}}{\varepsilon^{3}}\left(E p_{1}-p_{2}\right) b^{2} .
\end{aligned}
$$

The coefficients $A_{1}-A_{2}$ being quite lengthy and not needed in the discussion of overstability, have not been written here. Since $\sigma_{1}$ is real for overstability, the three values of $C_{1}\left(=\sigma_{1}^{2}\right)$ are positive. The product of the roots of (47) is $-A_{0} / A_{3}$ and if this is to be positive, then $A_{0}$ must be negative since $A_{3}$ is positive.
It is clear from (49) that $A_{0}$ is always positive if

$$
\begin{gathered}
E p_{1}>p_{2}, \quad \text { and }\left(\frac{E p_{1}}{P^{3}}+\frac{2 Q_{1}}{\varepsilon^{2} P}\right) \\
>\frac{U(b-3)^{2}}{\varepsilon},
\end{gathered}
$$

which implies that

$$
\begin{aligned}
& \kappa<\left[\varepsilon+(1-\varepsilon) \frac{\rho_{S} C_{S}}{\rho_{0} C}\right] \eta \text { and } \kappa \\
& <\frac{\left[\varepsilon+(1-\varepsilon) \frac{\rho_{S} C_{S}}{\rho_{0} C}\right] \eta}{P^{3}\left[\left(\frac{v_{0}}{v}\right)^{2} \varepsilon(b-3)^{2}-\frac{\mu_{e} H^{2} d^{2}}{2 \varepsilon^{2} \rho_{0} v \eta \pi^{3} P}\right]}
\end{aligned}
$$

The inequalities (51) are, therefore, the sufficient conditions for the non-existence of overstability, the violation of which does not necessarily imply the occurrence of overstability.

\section{Conclusions}

The thermal convection of a plasma in porous medium in the presence of finite Larmor radius effect is considered in the present paper. The main conclusions from the analysis of this paper are as follows:

- It is found that the finite Larmor radius and magnetic field introduce oscillatory modes in the system which were nonexistent in their absence.

- It is observed that the system is stable for $\frac{g \alpha \kappa}{v \beta} \leq \frac{27 \pi^{4}}{4}$ and under the condition $\frac{g \alpha \kappa}{v \beta}>\frac{27 \pi^{4}}{4}$, the system becomes unstable.

- For the case of stationary convection, finite Larmor radius has stabilizing effect on the system.

- It is also observed for the case of stationary convection that in the presence of finite Larmor radius effect, the magnetic field has a stabilizing (or destabilizing) effect and the medium permeability has a destabilizing (or stabilizing) effect under certain conditions whereas in the absence of finite Larmor radius effect, the magnetic field and the medium 
permeability have stabilizing and destabilizing effects, respectively.

- The case of overstability is also considered. The conditions

$$
\begin{aligned}
& \kappa<\left[\varepsilon+(1-\varepsilon) \frac{\rho_{S} C_{S}}{\rho_{0} C}\right] \eta \text { and } \kappa \\
& <\frac{\left[\varepsilon+(1-\varepsilon) \frac{\rho_{S} C_{S}}{\rho_{0} C}\right] \eta}{P^{3}\left[\left(\frac{v_{0}}{v}\right)^{2} \varepsilon(b-3)^{2}-\frac{\mu_{e} H^{2} d^{2}}{2 \varepsilon^{2} \rho_{0} v \eta \pi^{3} P}\right]}
\end{aligned}
$$

are the sufficient conditions for the nonexistence of overstability, the violation of which does not necessarily imply the occurrence of overstability.

\section{References}

[1] Chandrasekhar, S., Hydrodynamic and Hydromagnetic Stability, Dover Publication, New York, 1981.

[2] Jukes, J.D., Gravitational resistive instabilities in plasma with finite Larmor radius, Phys. Fluids, Vol. 7, 1964, pp. 52-58.

[3] Vandakurov, Ju. V., On the theory of stability of a plasma in a strong longitudinal magnetic field, variable along the axis of symmetry, Prikl. Mat. Mekh., Vol. 28, 1964, pp. 69-74.

[4] Sharma, R.C. and Prakash, K., Thermal instability in plasma with finite Larmor radius, Z. Naturforsch., Vol. 30a, 1975, pp. 461-465.

[5] Melchior, H. and Popowich, M., Effect of the finite ion Larmor radius on the KelvinHelmholtz instability, Phys. Fluids, Vol. 11, 1968, pp. 458-465.

[6] Singh, S. and Hans, H., Magnetic viscosity and the superposed fluids in a magnetic field, Nucl. Fusion, Vol. 6, 1966, pp. 6-11.

[7] Sharma, R.C., Finite Larmor radius and Hall effects on thermal instability of a rotating plasma, Phys. Fluids, Vol. 15, 1972, pp. 18221826.

[8] Joseph, D.D., Stability of Fluid Motions, Vol. 1 and 2, Springer-Verlag, Berlin, 1976.
[9] Lapwood, E.R., Convection of a fluid in a porous medium, Proc. Camb. Soc., Vol 44, 1948, pp. 508-554.

[10] Wooding, R.A., Rayleigh instability of a thermal boundary layer in flow through a porous medium, J. Fluid Mech., Vol. 9, 1960, pp. 183-192.

[11] Roberts, K.V. and Taylor, J.B., Magnetohydrodynamics equations for finite Larmor radius, Phys Rev. Lett., Vol. 8, 1962, pp. 197-198.

[12] Rosenbluth, M.N., Krall, N. and Rostoker, N., Finite Larmor radius stabilization of "weakly" unstable confined plasmas, Nucl. Fusion Suppl., Pt. 1, 1962, pp. 143-150.

[13] Hernegger, F., Effect of collisions and gyroviscosity on gravitational instability in a two-component plasma, J. Plasma Phys., Vol. 8, 1972, pp. 393-400.

[14] Sharma, R.C., Gravitational instability of a rotating plasma, Astrophys. Space Sci., Vol. 29, 1974, L1-L4.

[15] Ariel, P.D., Effect of Finite Larmor radius on the gravitational instability of a conducting plasma layer of finite thickness surrounded by a non-conducting matter, Astrophys. Space Sci., Vol. 141, 1988, pp. 141-149.

[16] Vaghela, D.S. and Chhajlani, R.K., Magnetogravitational stability of resistive plasma through porous medium with thermal conduction and FLR corrections, Contrib. Plasma Phys., Vol. 29, 1989, pp. 77-89.

[17] Bhatia, P.K. and Chhonkar, R.P.S., Larmor Radius effects on the instability of a rotating layer of a self-gravitating plasma, Astrophys. Space Sci., Vol. 115, 1985, pp. 327344.

[18] Vyas, M.K. and Chhajlani, R.K., Influence of finite Larmor radius with finite electrical and thermal conductivities on the gravitational instability of a magnetized rotating plasma through a porous medium, Contrib. Plasma Phys., Vol. 30, 1990, pp. 315328. 
[19] Kaothekar, S. and Chhajlani, R.K. Jeans instability of self-gravitating rotating radiative plasma with finite Larmor radius corrections, $J$. Phys. Conf. Series, 534, 012065, 2014.

[20] Spiegel, E.A., Convective instability in a compressible atmosphere, Astrophys. J., Vol. 141, 1965, pp. 1068-1070.

\section{Creative Commons Attribution License 4.0 (Attribution 4.0 International, CC BY 4.0)}

This article is published under the terms of the Creative Commons Attribution License 4.0

https://creativecommons.org/licenses/by/4.0/deed.en US 